\title{
Prenatal Stress and its effects of human cognition, behavior and psychopathology: A review of the literature
}

\author{
Mariano Enrique Ramborger ${ }^{1,2}$, María Aurelia Zorrilla Zubilete ${ }^{2,3 \dagger}$ and Gabriela Beatriz Acosta ${ }^{1,3 *}$ \\ ${ }^{1}$ Instituto de Investigaciones Farmacológicas (ININFA), Consejo Nacional de Investigaciones Científicas y Técnicas (CONICET), Facultad de Farmacia y \\ Bioquímica, Universidad de Buenos Aires (UBA), Argentina \\ ${ }^{2}$ Centro de Estudios Farmacológicos y Botánicos (CEFYBO - CONICET), Argentina \\ ${ }^{3}$ Departamento de Farmacología, Facultad de Medicina, Universidad de Buenos Aires, Argentina \\ ${ }^{\dagger}$ Decease
}

\begin{abstract}
Stress plays a fundamental role in the etiology and evolution of diseases and disorders both in the early stages of development and the adult life. Neuropsychiatric disorders such as depression, autism, ADHD and schizophrenia have a complex etiology, which is influenced by early experiences that affect the vulnerability of the individual.

During early intrauterine and postnatal life, the developing organism undergoes complex physiological changes, and even small disturbances can have far-reaching consequences which can be observed even into adulthood. Currently, understanding the factors that may be affecting the organism during development is a challenge. Among the effects induced by Prenatal Stress we can mention a dysregulation of the HPA axis, an alteration of the circadian rhythm, a reduction of the hippocampal plasticity and the capacity of learning and memory among others.

The effects of Prenatal Stress are widely studied in laboratory animals, but systematic research on human subjects is comparatively scarce. While studies in humans suffer from certain methodological limitations, they are currently the only way to explore some of the most complex, long-term interactions between antenatal stress and the behavioral and cognitive alterations of the offspring. This work aims to review studies about Prenatal Stress pertaining human subjects, and to explore some of the controversies arising on this field.
\end{abstract}

\section{Introduction}

Studies about the effects of Prenatal Stress part from the assumption that the developing organism is susceptible to fetal insults in utero which, in turn, lead to a series of long-lasting physiological and behavioral consequences $[1,2]$. The relationship between maternal stress during pregnancy and its effects on the child has been the subject of numerous studies both in humans and with the aid of animal models [3].

Although animal models allow for a greater degree of flexibility, human studies have some advantages over them. They permit to study the consequences of Prenatal Stress on human health and development without the need of developing often complex models for human behavior and physiology. Moreover, the resulting outcome may be closer to the average human situation, given that the stressors ensue in a fortuitous manner as opposed to the controlled way in which they administered in an experimental setting. Finally, the studies in humans bring a good deal of new variables to take into account, such as different coping strategies, the presence of support networks, the ability to self-report perceived stress, and the introduction of diverse contexts and life events $[4,5]$. While this comprises an increased number of confounding variables, it allows studying certain interactions that the animal models do not currently permit.

Most of the works centered in the effects of Prenatal Stress in humans suffer, however, from severe limitations. It is difficult to standardize the stressors suffered by the mothers, since the intensity and specific gestational period of the insult $[5,6]$ may result in different outcomes.
Consequently, many investigations pertaining humans choose to take a retrospective approach, taking far-reaching distressing situations, such as natural or man-made disasters as stress paradigms that allow for a certain degree of randomization [5,7]. However, maternal selfreports about prenatal anxiety may not be entirely reliable, especially when their children have developmental problems or disorders, as they may exaggerate the stress they felt during pregnancy [8].

A way to avoid this potential bias is to take a prospective approach. This involves follow-up studies of pregnant women under daily life stress $[9,10]$. After birth, different characteristics of their offspring are evaluated and examined, considering the degree of stress their mothers suffered during pregnancy.

Some articles measure maternal stress with questionnaires and self-reports, while other ones incorporate non-invasive physiological measures, such as salivary cortisol $[11,12]$.

This work aims to review human studies focusing on the impact of Prenatal Stress on the offspring, particularly those relating to potential

Correspondence to: Dra Gabriela B. Acosta, Instituto de Investigaciones Farmacológicas (ININFA) CONICET-UBA Junín 956, 5 piso C1113AAD, Buenos Aires, Argentina, Tel: 0054-11-4961-5949/6784, E-mail: gacosta@ffyb.uba.ar

Key words: Prenatal Stress, human studies, hypothalamic-pituitary-adrenal axis, neurodevelopment

Received: January 20, 2018; Accepted: February 15, 2018; Published: February 19,2018 

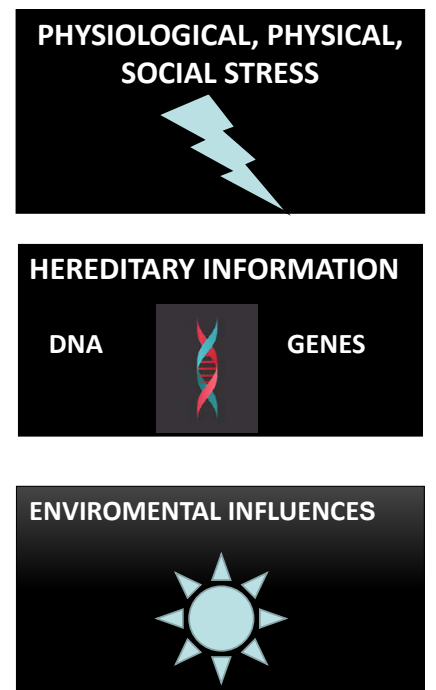

\section{PRENATAL STRESS}

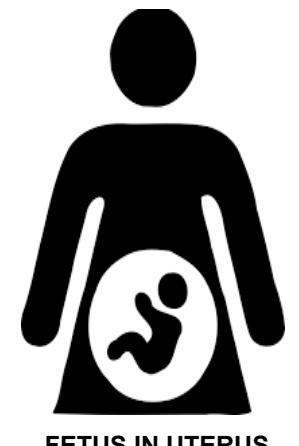

FETUS IN UTERUS

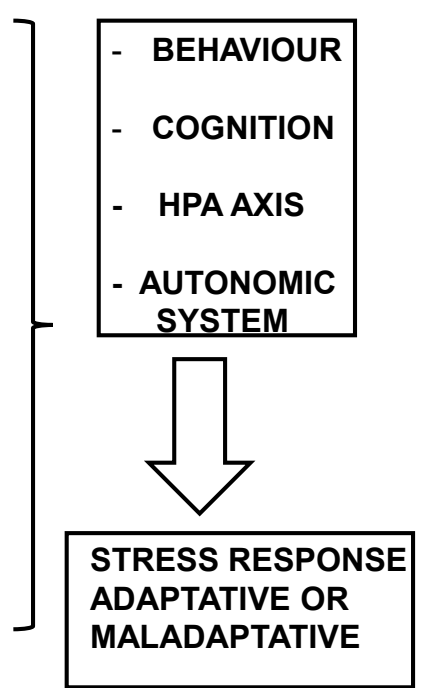

Figure 1. Physiological and pathological responses to stress. The resilience or vulnerability of an individual to situations of stress in adulthood.

behavioral and emotional problems, psychopathologies and cognitive functions.

\section{Studies in humans}

While classic research papers have focused on retrospective correlations between Prenatal Stress and child outcomes [5,13]. Some recent works opt for prospective studies, which allow a closer examination of the maternal stressors and the gestational process $[14,15]$.

Research on humans has focused mainly on the potential behavioral and emotional problems related to Prenatal Stress, as well as its connection to certain psychopathologies, particularly Attention Deficit Hyperactivity Disorder (ADHD). However, we observe two controversial issues that remain unanswered. The first one is related to the timing of the stressors along the gestational period, particularly regarding if the trimester in which the mother experiences elevated levels of distress is related to different postnatal outcomes. The second issue is a consequence of working with human subjects, and relates to whether the effects of the Prenatal Stress on the offspring, depend more on the objective qualities of the stressors, like intensity, frequency and duration, or subject-related variables, such as coping skills and the perception of the stress experience.

Most likely there is not a clear-cut general answer to the aforementioned controversies. Both objective and perceived stress may exert different influences in the development of the offspring, and there may be distinct effects depending on the time of the stressor. While not many investigations focus on these issues of interest, they are often taken as variables in many investigations. The controversies are featured mainly in works pertaining the relationship between Prenatal Stress, behavioral and emotional problems, psychopathology and intelligence.

\section{Behavioral and emotional problems}

An early work focused on pregnant Scottish Women subjected to different types of objective stress, such as illness or accidents, with and without the presence of interpersonal stress. Only the chronic interpersonal stressors were related to behavioral disturbance in their offspring [13]. Other studies have reported that toddlers whose mothers had high anxiety during pregnancy cry more often and have increased risk to develop habit disorders $[16,17]$.

A few of the most important works related to Prenatal Stress in humans are based on the Avon Longitudinal Study of Parents and Children (ALSPAC). The ALPSAC is an ambitious study aiming to gather data from all the women who were pregnant during 1991 and 1992 in the city of Avon, England, as well their partners and their children. The main objective of the ALSPAC was to study the interaction of the genes and the environment, and their consequences on the offspring health and development [18]. Works with the ALSPAC sample have found that high levels of anxiety during late pregnancy were correlated with increased emotional and behavioral problems at 4 and 6 years regardless of postnatal factors $[14,19]$ and more sleep problems at 18 and 30 months [20]. Moreover, high maternal anxiety during midpregnancy has been associated with high anxiety as reported by the 8 and 9 years old offspring [21].

A study with Dutch Women exposed to high anxiety during early pregnancy, found that their perceived stress predicted difficult behavior, trouble to adapt to new contexts, and crying in their toddlers. Anxiety related to pregnancy was found to be related to these conduct problems, but daily hassles were not [22]. Another paper supports the hypothesis that perceived stress and pregnancy specific-anxiety are good predictors of more externalizing behaviors and difficult temperaments in 27 months old children [23].

Regarding objective stress, it has been suggested that exposure to a high amount of Prenatal Stressful events, results in children with increased fearfulness, even after controlling for postnatal confounding variables [1]. A recent investigation with low-income MexicanAmerican mothers noted that children exposed to elevated prenatal maternal anxiety were more likely to display higher temperamental negativity, and to engage in less self-comforting behaviors [10]. In summary, these studies suggest that high anxiety during pregnancy heightens the chances of behavioral and emotional problems in childhood. Perceived stress of the mother, and especially pregnancyspecific anxiety, seems to be of particular importance. However, more prospective studies are needed to assess if the timing and intensity 
of the stressors are relevant to problematic conducts and impaired emotional regulation. Researching the potential protective effects of mild Prenatal Stress is an interesting yet underexplored line of work.

\section{Attention Deficit Hyperactivity Disorder (ADHD)}

In humans, many works have studied the link between Prenatal Stress and psychopathology, with varying degrees of success. Among the different disorders taken into account, ADHD has been the main focus of studies about antenatal maternal distress.

On some of the previously cited articles [14,19], it was reported that high levels of maternal anxiety during late pregnancy were associated with increased risk of hyperactivity and inattention. Another paper observed increased movement on the fetuses of women with high anxiety, as well as increased toddler motor activity, as well as being "too active" as reported by their mothers [16]. Another study found out that maternal antenatal stress, was a better predictor of difficulties with attention regulation and externalizing problems in the offspring, than the presence of objective stressors. They also underscore the importance of pregnancy-specific anxiety (like the fear of having a handicapped child) on the offspring temperamental and behavioral development [15]. The association with pregnancy-specific anxiety and poorer emotional and attentional regulation has also been found in a sample of financially stable women with wanted pregnancies and no exposure to traumatic stressors [24].

In contrast, a previously cited article notes that children in a vulnerable, low-income migrant population, displayed increased orienting behavior at 12 weeks [10]. Further studies are needed to determine if this result is an isolated anomaly or there is indeed a protective effect of Prenatal Stress on orienting behavior.

Subjective self-reported maternal stress during mid-pregnancy was also associated with ADHD symptoms in 8 and 9 year olds [21]. Additionally, a study pertaining the three-way relation between smokings, Prenatal Stress and ADHD, argues that perceived stress, up to the first half of pregnancy, is linked with the development of ADHD symptoms, independently of smoking [25]. Nevertheless, the former study suffered from an admittedly low sample of children who fulfilled the ADHD criteria. Another investigation reported that mothers who suffered more objective stressful life events, like the death of a relative, or financial problems, were more likely to have children with ADHD symptoms, and boys with autistic traits [26].

Recent studies have tried to solve the problem of small samples that diminished the statistical power of previous studies. In a large sample of children with ADHD, infants (specially boys) of women who suffered severe objective stressors during pregnancy, such as physical abuse or the death of a close relative, had more intense ADHD symptoms, than those with mothers who suffered mild to no stress during pregnancy [27]. Boys were also more vulnerable to ADHD symptoms in another prospective, longitudinal study. They were twice as likely to have clinically-relevant ADHD symptoms, if their mothers suffered life events that they evaluated as extremely stressful during their second pregnancy trimester [28]. For girls, severe stressful events were only predictive of ADHD if they ensued during the third trimester of pregnancy.

The articles previously cited concur in that there is a strong link between Prenatal Stress, and ADHD symptoms. This may either be a cause or a consequence of the fact that it's the most studied disorder in its relation to antenatal anxiety. More studies are needed to asseverate if the timing and the nature of the stress (subjective or objective) are a factor on its incidence of ADHD symptoms in the offspring.

\section{Other psychopathologies}

The relationship between Prenatal Stress and psychopathologies other than ADHD has also been studied, although to a lesser degree.

One investigation [8] retroactively analyzed the timing of the maternal stressors in samples constituted of children with autism, Down syndrome and a control group. It was found that mothers of autistic children reported a greater quantity of stressors during pregnancy than the Down syndrome group, and both groups reported more stressors than the control group. However, as the authors acknowledge, the retrospective nature of this study doesn't account for possible group biases when reporting past stressors. Additionally, autistic children whose mothers were subjected to stress during mild-pregnancy were more to likely to lack language than those who suffered it during early or late pregnancy. The timing of the stressors has also been the focus of another work [26], which used a series of storms that affected Louisiana between 1985 and 1995 as a natural experiment. The results suggest that middle and late pregnancy could be sensitive gestational periods in which severe stressors may influence the prevalence of autistic disorder. A prospective investigation reported a link between nonspecific stressors and autistic traits in 2-years old children, although it does not include data about the severity or timing of the stressors [26]. On the other hand, a recent study did not find a significant association between the loss of a close relative during pregnancy and autism in the offspring. However, this work did not measure the maternal emotional state during gestation [30], which may be associated with an increased risk of autism [31].

Another psychopathology that has received some degree of attention, pertaining its relationship with Prenatal Stress, is schizophrenia. An early work suggests a small increase in the risk for schizophrenia when the mother is exposed to severe stress, such as war [32]. A prospective study suggests that an acute stressor, like the death of a relative, may increment the chance of developing schizophrenia, but only in families without history of mental illness [33]. Yet another prospective investigation, reports that the risk for schizophrenia doubled for the mothers who were exposed to war during their second trimester of pregnancy [34].

While it's highly unlikely that Prenatal Stress has a causal effect in the chance of developing autism or schizophrenia, it cannot be discounted that it may contribute to increase the chances of expressing either pathology, by mechanisms that are yet unknown. Prospective studies focusing on both the timing and type of stressors, as well as the maternal emotional state during pregnancy would serve to asses if there is an actual link between antenatal stress and the aforementioned pathologies.

It is unlikely that human studies alone would be enough to offer an explanatory hypothesis for the way antenatal stress contributes to psychopathologies. While studies using laboratory animals bring new methods and tools to study this influence, the validity of animal models for schizophrenia and autism are a matter of debate and constant revision [35]. Nevertheless, advances in the animal model of neuropsychiatric disorders may greatly benefit our understanding of the effects of Prenatal Stress.

\section{Intelligence}

In 1998 a succession of Ice Storms ensued in Quebec, Canada. The freezing rain caused widespread damage. Telephone and electricity lines went down, leaving people without service and hurting business, homes were destroyed, and 27 people lost their lives. A series of studies 
pertaining exposure to objective Prenatal Stress were performed on the mothers who suffered the Ice Storm, and their offspring.

A classic investigation evaluated the intellectual and linguistic abilities of two years old toddlers whose mothers were subjected to the Ice Storm. Their performance was negatively correlated with the degree of objective Prenatal Stress exposure. They spoke and understood fewer words, and had a lower score on the mental development index than toddlers who suffered milder to none Prenatal Stress. Toddlers exposed to the storm during the first or second gestational trimester had increased chances to have lower scores on the intellectual abilities scale [5]. Further analysis on the same population reveals that objective stress has a greater impact on cognitive abilities than perceived stress, and that early pregnancy stress predicts poorer cognitive function, than late pregnancy stress [36]. Three years later, a follow-up study was conducted to determine whether the effects of Prenatal Stress were still associated with intelligence and language skills at five years old. Again, elevated objective Prenatal Stress predicted lower performance on linguistic and cognitive tasks. Interestingly, children who suffered moderated Prenatal Stress may perform better than those whose mothers were exposed to either high or low stress during pregnancy [37].

Different articles have reported similar results on different populations. A study on Californian women found that high pregnancy-specific anxiety during early pregnancy is associated with poorer mental development at one year of life. It is worth noting than toddlers whose mothers were exposed to low levels of stress during early pregnancy, and high levels of stress during late pregnancy, had the best performance [38]. An investigation with Londoners found that an elevated number of objective stressors during pregnancy predict slower mental development. In contrast with the Ice Storm studies, subjective stress was also significantly associated with mental development, and relationship strain was the best predictor [9]. Consequently, another work reported that early Prenatal Stress was negatively correlated with children's school marks at six years old [39]. High anxiety during the first half of pregnancy may also be associated with performance in cognitive tasks at ages 14 and 15, although it may be due to increased impulsivity and difficult to maintain attention [1]. In contrast to the previous studies, a recent work has reported no associations between Prenatal Stress and Intelligence quotient [27]. However, given that all the children of the sample were diagnosed with ADHD, this may not be the case for the general population.

There is a clear consensus that stressors, especially objective stressors during early and mid-pregnancy have a far greater impact in mental development than during the latter half. While evidence supports that antenatal anxiety has an effect on intelligence and language skills, more human studies are needed to determine by which mechanisms disrupt development. Little literature so far inquiries about the effects of Prenatal Stress in the cognitive performance of adolescent and adults. While such studies involve significant constraints, they may help to explain, with proper control for postnatal variables, the reasons of the diminished performance. In summary, the aforementioned studies suggest that Prenatal Stress may affect mental development through decreased self-regulation capacity, impoverished attention or increased impulsivity; further work is needed to determine in which specific ways it affects the executive functions.

\section{The HPA axis}

While the previous sections of this work have focused in the possible effects of antenatal stress, the mechanisms by which these changes take place have yet to be thoroughly described. Several studies in both animals and humans have pointed out the importance of the HPA Axis on the study of the effects of Prenatal Stress. While a detailed review of the HPA axis involvement in the intergenerational transmission of stress is beyond the scope of the present paper, we highlight some works that have explored this relationship in humans.

It has been proposed that Prenatal Stress has a programming effect in the HPA axis, altering it as a predictive adaptive response to its post-natal environment [40]. The exact mechanisms by which these environmental cues are relayed is still being studied, but evidence suggests that's signaled by hormones, particularly glucocorticoids.

Glucocorticoids are typically secreted in response to stressors. An excessive concentration of glucocorticoids can have deleterious effects on the developing organism $[41,42]$ and it could permanently affect the expression of Glucocorticoid receptors [43] which are vital for the negative feedback circuit that inhibits Glucocorticoid secretion [44].

The 11ß-HSD2 or Placental 11ß-Hydroxy Steroid Dehydrogenase Type II, is an enzyme present in the placenta which protects the fetus from cortisol, transforming it in cortisone [41]. A high amount of anxiety during the pregnancy may impair the placental protective function [45], leaving the fetus exposed to an excessive and potentially deleterious amount of cortisol.

It should be noted that the programming of the HPA axis in response to an environment perceived as hostile or dangerous, it's in principle an adaptive response. The negative effects of antenatal stress may come from a mismatch between the early life and adulthood environments [46] given that the organism suffered modifications that are no longer relevant and may be maladaptive to the actual living conditions of the mature individual.

The programming hypothesis has been thoroughly studied in animals, where it's possible both to control the maternal stressors, and even design animals that lack 11ß-HSD2. Nevertheless, the HPA activity and the effects of high cortisol during pregnancy have also been studied in humans through salivary cortisol and placental analysis.

A study using salivary cortisol as a biomarker for stress, suggests that the HPA axis response to stress varies along the course of gestation, with increased maternal cortisol concentrations during late pregnancy [47]. This change in stress reactivity may support the idea that timing of stressors is relevant to the offspring vulnerability to certain disorders or disturbances. Maternal stress during pregnancy has been linked to an altered HPA axis regulation in the offspring, leading to increased basal levels of cortisol $[48,49]$, as well releasing more cortisol when exposed to stressors such as the first day of school [23], a social stress test [50] and the painful stimulation of a heel-stick blood draw [51].

Animal studies can give us a clearer perspective about the ways in which the HPA axis undergoes modifications in response to Prenatal Stress, but human studies, especially prospective ones, are particularly well-suited to assess the long-term consequences of antenatal stress, and it is interaction with different vital circumstances. Research in humans pertaining this subject is indeed bound to face methodological complications, but it also brings exciting possibilities.

\section{Conclusions}

The effect of Prenatal Stress in humans comprises a study field with many different fronts. While it's generally accepted that antenatal insults influence diverse variables, such as intelligence, behavioral tendencies, stress vulnerability and the risk to develop certain pathologies, the degree of impact of Prenatal Stress is under constant revision. 
High antenatal anxiety has been consistently associated with a higher chance to express ADHD, and, to a lesser degree, autism and schizophrenia. The latter disorders need more prospective studies to determine if the proposed link goes beyond correlation, and comparative studies in combination with the animal model to elaborate explicative hypothesis about the mechanisms underlying the influence of Prenatal Stress in the onset of psychopathology. Particularly, more prospective human-based investigations incorporating physiological variables such as cortisol concentrations (both in the mother during pregnancy and the child when confronted with a stressor) are needed to properly measure the far-reaching effects of Prenatal Stress in humans.

The same is true to discern the way in which antenatal objective stressors disrupt development, as it has been linked to impaired intelligence and language skills. Consensus suggests that both high and a very low amount of stress during pregnancy are associated with poorer performance in tests regarding intelligence and language skills. This effect seems more pronounced when the stressors ensue during early and mid-pregnancy.

Prenatal Stress may also enhance behavioral and emotional problems in the offspring, but more work is needed to determine if the timing of the stressors is a relevant factor. Additionally, pregnancyspecific anxiety may be a preponderant stressor when it comes to influencing emotional reactivity and behavioral tendencies.

Despite its shortcomings, studies in humans present themselves as a privileged paradigm to study the complex interrelationship between antenatal stressors, the associated maternal response, and the resulting changes in the offspring. While it's unlikely that it will be able to provide causal explanations by itself, it appears as a vital complement for animal investigations. Human studies are a particularly important in pathologies and behaviors which cannot be studied in animals, for the lack of reliable paradigms.

An advance in the knowledge pertaining the effects of Prenatal Stress in humans may open an exciting new field of prevention and early care for mothers, particularly ones exposed to stressful situations. More work is needed to ascertain the specific effects of high antenatal anxiety, and, past that, we may start to see applied research oriented at diminishing the potential noxious effects of Prenatal Stress in humans.

\section{Acknowledgements}

Dedicated to the loving memory of Dr Maria Aurelia Zorrilla Zubilete, who died in September of 2017, after battling a serious illness. This work was supported by grants PIP No 0409 from National Scientific and Technologic Research Council (CONICET) to GBA. GBA is member of CONICET.

\section{Conflict of interest statement}

The authors report no conflicts of interest. The authors alone are responsible for the content and writing of the manuscript.

\section{References}

1. Van den Bergh BR, Mulder EJ, Mennes M, Glover V (2005) Antenatal maternal anxiety and stress and the neurobehavioural development of the fetus and child: links and possible mechanisms. A review. Neurosci Biobehav Rev 29: 237-258. [Crossref]

2. Glover V (2011) Annual research review: Prenatal Stress and the origins of psychopathology: an evolutionary perspective. J Child Psychol Psychiatry 52: 356367. [Crossref]

3. Beydoun H, Saftlas AF (2008) Physical and mental health outcomes of prenatal maternal stress in human and animal studies: a review of recent evidence. Paediatr Perinat Epidemiol 22: 438-466. [Crossref]
4. Joffe JM (2013) Prenatal Determinants of Behaviour: International Series of Monographs in Experimental Psychology 7: Elsevier.

5. Laplante DP1, Barr RG, Brunet A, Galbaud du Fort G, Meaney ML, et al. (2004) Stress during pregnancy affects general intellectual and language functioning in human toddlers. Pediatr Res 56: 400-410. [Crossref]

6. Davis EP, Sandman CA (2010) The timing of prenatal exposure to maternal cortisol and psychosocial stress is associated with human infant cognitive development. Child Development 81: 131-148. [Crossref]

7. Kinney DK, Miller AM, Crowley DJ, Huang E, Gerber E. (2008) Autism prevalence following prenatal exposure to hurricanes and tropical storms in Louisiana. J Autism Dev Disord 38: 481-488. [Crossref]

8. Beversdorf DQ, Manning SE, Hillier A, Anderson SL, Nordgren RE, et al. (2005) Timing of Prenatal Stressors and autism. J Autism Dev Disord 35: 471-478. [Crossref]

9. Bergman K1, Sarkar P, O'Connor TG, Modi N, Glover V (2007) Maternal Stress During Pregnancy Predicts Cognitive Ability and Fearfulness in Infancy. $J$ Am Acad Child Adolesc Psychiatry 46: 1454-1463. [Crossref]

10. Lin B, Crnic KA, Luecken LJ, Gonzales NA (2014) Maternal Prenatal Stress and infan regulatory capacity in Mexican Americans. Infant Behav Dev 37: 571-582. [Crossref]

11. Yehuda R, Engel SM, Brand SR, Seckl J, Marcus SM, et al. (2005) Transgenerational effects of posttraumatic stress disorder in babies of mothers exposed to the World Trade Center attacks during pregnancy. J Clin Endocrinol Metab 90: 4115-4118. [Crossref]

12. Davis EP, Glynn LM, Schetter CD, Hobel C, Chicz-Demet A, et al. (2007) Prenatal exposure to maternal depression and cortisol influences infant temperament. J Am Acad Child Adolesc Psychiatry 46: 737-746. [Crossref]

13. Stott DH (1973) Follow-up Study from Birth of the Effects of Prenatal Stresses. Dev Med Child Neurol 15: 770-787. [Crossref]

14. O'Connor TG, Heron J, Golding J, Beveridge M, Glover V (2002) Maternal antenatal anxiety and children's behavioural/emotional problems at 4 years. Br J Psychiatry 180: 502-508. [Crossref]

15. Gutteling BM, de Weerth C, Willemsen-Swinkels SH, Huizink AC, Mulder EJ, et al. (2005) The effects of Prenatal Stress on temperament and problem behavior of 27-month-old toddlers. Eur Child Adolesc Psychiatry 14: 41-51. [Crossref]

16. Van den Bergh B (1990) The influence of maternal emotions during pregnancy on feta and neonatal behavior. Pre-and Perinatal Psychology Journal 5: 119-30.

17. Ward AJ (1991) Prenatal Stress and childhood psychopathology. Child Psychiatry Hum Dev 22: 97-110. [Crossref]

18. Golding J, Pembrey M, Jones R; ALSPAC Study Team (2001) ALSPAC-the Avon longitudinal study of parents and children. I. Study methodology. Paediatr Perinat Eepidemiol 15: 74-87. [Crossref]

19. O'Connor TG, Heron J, Golding J, Glover V; ALSPAC Study Team. (2003) Materna antenatal anxiety and behavioural/emotional problems in children: a test of a programming hypothesis. J Child Psychol Psychiatry 44: 1025-1036. [Crossref]

20. O'Connor TG, Caprariello P, Blackmore ER, Gregory AM, Glover V, et al. (2007) Prenatal mood disturbance predicts sleep problems in infancy and toddlerhood. Early Human Dev 83: 451-458. [Crossref]

21. Van den Bergh BR, Marcoen A (2004) High antenatal maternal anxiety is related to ADHD symptoms, externalizing problems, and anxiety in 8-and 9-year-olds. Child Dev 75: 1085-1097. [Crossref]

22. Huizink AC, de Medina PG, Mulder EJ, Visser GH, Buitelaar JK (2002) Psychological measures of Prenatal Stress as predictors of infant temperament. J Am Acad Child Adolesc Psychiatry 41: 1078-1085. [Crossref]

23. Gutteling BM, de Weerth C, Buitelaar JK (2005) Prenatal Stress and children's cortisol reaction to the first day of school. Psychoneuroendocrinology 30: 541-549. [Crossref]

24. DiPietro JA, Novak MF, Costigan KA, Atella LD, Reusing SP (2006) Materna psychological distress during pregnancy in relation to child development at age two. Child Dev 77: 573-587. [Crossref]

25. Rodriguez A, Bohlin G (2005) Are maternal smoking and stress during pregnancy related to ADHD symptoms in children? J Child Psychol Psychiatry 46: 246-254. [Crossref]

26. Ronald A, Pennell CE, Whitehouse AJ (2011) Prenatal maternal stress associated with ADHD and autistic traits in early childhood. Front Psychol 1: 223. [Crossref]

27. Grizenko N, Fortier MĖ, Gaudreau-Simard M, Jolicoeur C, Joober R (2015) The effect of maternal stress during pregnancy on IQ and ADHD symptomatology. J Can Acad Child Adolesc Psychiatry 24: 92. [Crossref] 
28. Zhu P, Hao JH, Tao RX, Huang K, Jiang XM, et al. (2015) Sex-specific and timedependent effects of Prenatal Stress on the early behavioral symptoms of ADHD: a longitudinal study in China. Eur Child Adolesc Psychiatry 24: 1139-1147. [Crossref]

29. Kinney DK, Miller AM, Crowley DJ, Huang E, Gerber E (2008) Autism Prevalence Following Prenatal Exposure to Hurricanes and Tropical Storms in Louisiana. J Autism Dev Disorder 38: 481-488. [Crossref]

30. Li J, Vestergaard M, Obel C, Christensen J, Precht DH, et al. (2009) A nationwide study on the risk of autism after Prenatal Stress exposure to maternal bereavement. Pediatrics 123: 1102-1107. [Crossref]

31. Zhang X, Lv CC, Tian J, Miao RJ, Xi W, et al. (2010) Prenatal and perinatal risk factors for autism in China. J Autism Dev Disord 40: 1311-1321. [Crossref]

32. Van Os J, Selten, JP (1998) Prenatal exposure to maternal stress and subsequent schizophrenia. The May 1940 invasion of The Netherlands. Br J Psychiatry 172: 324326. [Crossref]

33. Khashan AS1, Abel KM, McNamee R, Pedersen MG, Webb RT, et al. (2008) Higher risk of offspring schizophrenia following antenatal maternal exposure to severe adverse life events. Arch Gen Psychiatry 65: 146-152. [Crossref]

34. Malaspina D1, Corcoran C, Kleinhaus KR, Perrin MC, Fennig S, et al. (2008) Acute maternal stress in pregnancy and schizophrenia in offspring: a cohort prospective study. BMC Psychiatry 8: 71. [Crossref]

35. Patterson PH (2009) Immune involvement in schizophrenia and autism: etiology, pathology and animal models. Behav Brain Res 204: 313-321. [Crossref]

36. King S, Laplante DP (2005) The effects of prenatal maternal stress on children's cognitive development: Project Ice Storm. Stress 8: 35-45. [Crossref]

37. Laplante DP, Brunet A, Schmitz N, Ciampi A, King S (2008) Project Ice Storm: prenatal maternal stress affects cognitive and linguistic functioning in $5 \frac{1}{2}$-year-old children. $J$ Am Acad Child Adolesc Psychiatry 47: 1063-1072. [Crossref]

38. Davis EP, Sandman CA (2010) The timing of prenatal exposure to maternal cortisol and psychosocial stress is associated with human infant cognitive development. Child Dev 81: 131-148. [Crossref]

39. Niederhofer H, Reiter A (2004) Prenatal maternal stress, prenatal fetal movements and perinatal temperament factors influence behavior and school marks at the age of 6 years. Fetal Diagn Ther 19: 160-162. [Crossref]
40. Glover V, O'Connor TG, O’Donnell K (2010) Prenatal Stress and the programming of the HPA axis. Neurosci Biobehav Rev 35: 17-22. [Crossref]

41. Benediktsson R, Calder AA, Edwards CR, Seckl JR (1997) Placental 11ß-hydroxysteroid dehydrogenase: a key regulator of fetal glucocorticoid exposure. Clin Endocrinol (Oxf) 46: 161-166. [Crossref]

42. Carpenter T, Grecian SM, Reynolds RM (2017) Sex differences in early-life programming of the hypothalamic-pituitary-adrenal axis in humans suggest increased vulnerability in females: a systematic review. J Dev Orig Health Dis 8: 244-255. [Crossref]

43. Welberg LA, Seckl JR (2001) Prenatal Stress, glucocorticoids and the programming of the brain. J Neuroendocrinol 13: 113-128. [Crossref]

44. Herman JP, McKlveen JM, Solomon MB, Carvalho-Netto E, Myers B (2012) Neural regulation of the stress response: glucocorticoid feedback mechanisms. Braz J Med Biol Res 45: 292-298. [Crossref]

45. Glover V, Bergman K, Sarkar P, O’Connor TG (2009) Association between maternal and amniotic fluid cortisol is moderated by maternal anxiety. Psychoneuroendocrinology 34: 430-435. [Crossref]

46. Nederhof E, Schmidt MV (2012) Mismatch or cumulative stress: toward an integrated hypothesis of programming effects. Physiol Behav 106: 691-700. [Crossref]

47. Obel C, Hedegaard M, Henriksen TB, Secher NJ, Olsen J, et al. (2005) Stress and salivary cortisol during pregnancy. Psychoneuroendocrinology 30: 647-656. [Crossref]

48. O'Connor TG, Ben-Shlomo Y, Heron J, Golding J, Adams D, et al. (2005) Prenatal anxiety predicts individual differences in cortisol in pre-adolescent children. Biol Psychiatry 58: 211-217. [Crossref]

49. Van den Bergh BR, Van Calster B, Smits T, Van Huffel S, Lagae L (2008) Antenatal maternal anxiety is related to HPA-axis dysregulation and self-reported depressive symptoms in adolescence: a prospective study on the fetal origins of depressed mood. Neuropsychopharmacology 33:.536-545. [Crossref]

50. Entringer S, Kumsta R, Hellhammer DH, Wadhwa PD, Wüst S (2009) Prenatal exposure to maternal psychosocial stress and HPA axis regulation in young adults. Horm Behav 55: 292-298. [Crossref]

51. Davis EP, Glynn LM, Waffarn F, Sandman CA (2011) Prenatal maternal stress programs infant stress regulation. J Child Psychol Psychiatry 52: 119-129. [Crossref]

Copyright: (C2018 Ramborger ME. This is an open-access article distributed under the terms of the Creative Commons Attribution License, which permits unrestricted use, distribution, and reproduction in any medium, provided the original author and source are credited. 